\title{
Performance Comparison and Visualization with Different Computational Softwares for Predicting the Reservoir Pressure on Oil Production
}

\author{
W. Z. A. W. Muhamad', N. Alias², , M. N. M. Ibrahim³ ${ }^{3}$ H. F. S. Saipol ${ }^{4}$, A. K. Junoh \\ ${ }^{1,5}$ Institute of Engineering Mathematics, Faculty of Applied and Human Sciences, \\ Universiti Malaysia Perlis (UniMAP), Perlis, Malaysia. \\ ${ }^{2}$ Centre for Sustainable Nanomaterials, Ibnu Sina Institute for Scientific and Industrial Research, \\ Universiti Teknologi Malaysia, Johor, Malaysia. \\ ${ }^{3}$ School of Chemical Sciences, Universiti Sains Malaysia, Pulau Pinang, Malaysia. \\ ${ }^{4}$ Malaysia-Japan International Institute of Techology (MJIIT), Johor, Malaysia.
} ORCIDs: 0000-0001-5697-8196 (W. Z. W. A Muhamad), 0000-0002-1855-1375 (N. Alias), 0000-0002-6784-5775 (M. N. M. Ibrahim),
0000-0002-0580-2227 (H. F. S. Saipol), 0000-0002-7705-4707 (A. K. Junoh)

\begin{abstract}
This paper presents the performance comparison of some computation software for solving the boundary element method (BEM). BEM formulation is the numerical technique and high potential for solving the advance mathematical modeling to predict the production of oil well in arbitrarily shaped based on multiple leases reservoir. The limitation of data validation for ensuring that a program meets the accuracy of the mathematical modeling is considered as the research motivation of this paper. Thus, based on this limitation, there are three steps involved to validate the accuracy of the oil production simulation process. In the first step, identify the mathematical modeling based on partial differential equation (PDE) with Poisson-elliptic type to perform the BEM discretization. In the second step, implement the simulation of the 2D BEM discretization using COMSOL Multiphysic and MATLAB programming languages. In the last step, analyze the numerical performance indicators for both programming languages by using the validation of Fortran programming. The performance comparisons of numerical analysis are investigated in terms of percentage error, comparison graph and $2 \mathrm{D}$ visualization of pressure on oil production of multiple leases reservoir. According to the performance comparison, the structured programming in Fortran programming is the alternative software for implementing the accurate numerical simulation of BEM. As a conclusion, high-level language for numerical computation and numerical performance evaluation are satisfied to prove that Fortran is well suited for capturing the visualization of the production of oil well in arbitrarily shaped.
\end{abstract}

Keywords: Performance comparison, 2D visualization, COMSOL multiphysics, Matlab, Fortran, BEM, FDM, reservoir pressure.

\section{INTRODUCTION}

The world demand for oil continues to grow despite the unpredictable world economy. Hydrocarbon fuels stay approximately low-priced and sensible as compared to the other fuel sources such as nuclear and electricity [5]. Therefore, the exploration of new oil fields prompts drilling of new wells. When the expectation of hydrocarbon is commercially justified, drilling operations will be started. The well reservoir is ever drilled, the untapped gas sit in the upper portions of the reservoir inside the tiny pore spaces. The pressure and temperature are considered different from surface conditions

Based on the chronology of oil and gas research, various techniques and approaches have been applied to predict the productivity, estimation and identification of oil, gas and natural material extractions [1], [3]. The traditional methods to measure and inspect the productivity of oil wells, mainly consisted using test separator units with expansive influential, mechanical, electrical, piping, services, facilities and infrastructures [6],[23]. The traditional method can be improved in terms of some parameter identification by implementing the mathematical model as an instrument to predict the productivity of oil wells.

References [2], [3], [6], [10], and [14] presented mathematical model on a regularly shaped reservoirs. Meanwhile, these authors [8], [11], and [19] focused on irregular shaped reservoirs. The productivity forecast of irregularly shaped reservoirs of oil wells is a difficult problem because the productions are unequal; the productions of outer fractures are higher than middle fractures; and the pressure in the well bore shows an uneven distribution. BEM has been known to be a generalized method solves irregular shaped reservoirs [17]. Thus, [4], [8], [11] used BEM modelling for predicting the productivity of oil wells arranged in complex configurations within irregularly shaped reservoirs. While [12] focused on the governing the production of in a multiple leases reservoir using BEM. Limitation of the previous research is lack of validation process of determining the accuracy of mathematical modelling and simulation. Model verification and validation by the existing and simulated data is necessary to ensure that the numerical scheme and simulation program are correct [1], [11] and [13]. Thus, the motivation of this paper is to compare some programming languages and visualization of the BEM formulation. The performance evaluations are needed to determine the modelling and simulation numerically. 


\section{BEM FOR WITH POISSON-ELLIPTIC EQUATION}

Reference [16] has used PDE with parabolic, hyperbolic and elliptic types and solving the numerical method based on analytical approach. Reference [15] believed that Poisson equation is particularly well suited for solving electrostatic potential problems. BEM for with Poisson-elliptic equation is developed by the transformation of Darcy's Law into the continuous equation. Recognize a hypothetical twodimensional homogeneous reservoir, $S$ having sources, NSS and sink placed randomly within an arbitrary shaped reservoir. The following assumption was used in establishing the theory [5] and [19].

a) The reservoir is considered as steady-state flow with pressure of the reservoir above bubble points that were under-saturated condition.

b) A small constant compressibility and constant viscosity is flowing in the system with single phase fluid.

c) The flow system is two dimensional ( $x$ and $y$ directions).

d) The reservoir has a finite boundary and a uniform thickness.

e) Gravitational effects are inconsequential.

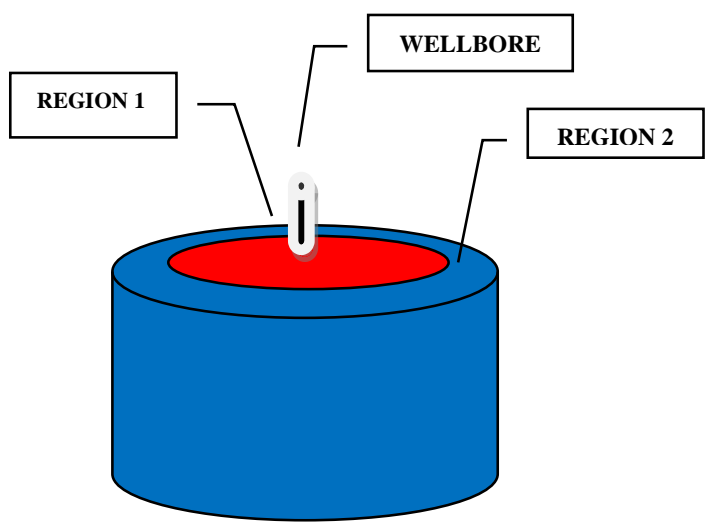

Fig. 1 A simple model of reservoir

[20] and [21] shown the important role of PDE with elliptic type for solving interdisciplinary problem. This paper emphases Poisson-elliptic differential equation to perform the pressure distribution in the reservoir by impressive the conditions and assumption that used in establishing theory. The governing equation is given by:

$\frac{\partial^{2} p}{\partial X^{2}}+\frac{\partial^{2} p}{\partial Y^{2}}+\frac{\mu}{k} \sum_{m=1}^{N S S} q_{m} \delta\left(X-X_{m}, Y-Y_{m}\right)=0$

where $p$ is pressure, $\mu$ is the dynamic viscosity of the fluid, $k$ is the permeability, $q_{m}$ is the flow rate of the $m^{t h}$ well per unit area (positive for injectors and negative producers), $\delta$ is the Dirac delta function, $X, Y$ are the coordinates axes, and $X_{m}, Y_{m}$ are the coordinates of the $m^{\text {th }}$ source and/or sink where $m$ goes for 1 to NSS. The section III will discussed the numerical techniques used to solve the Poisson-elliptic (1).

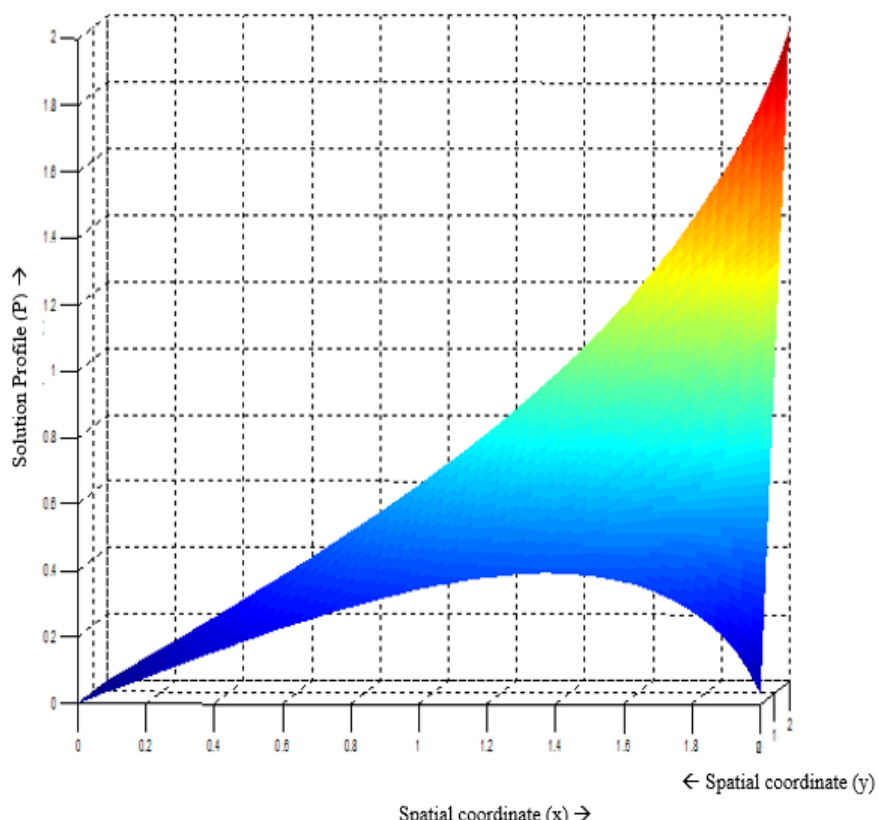

(a)

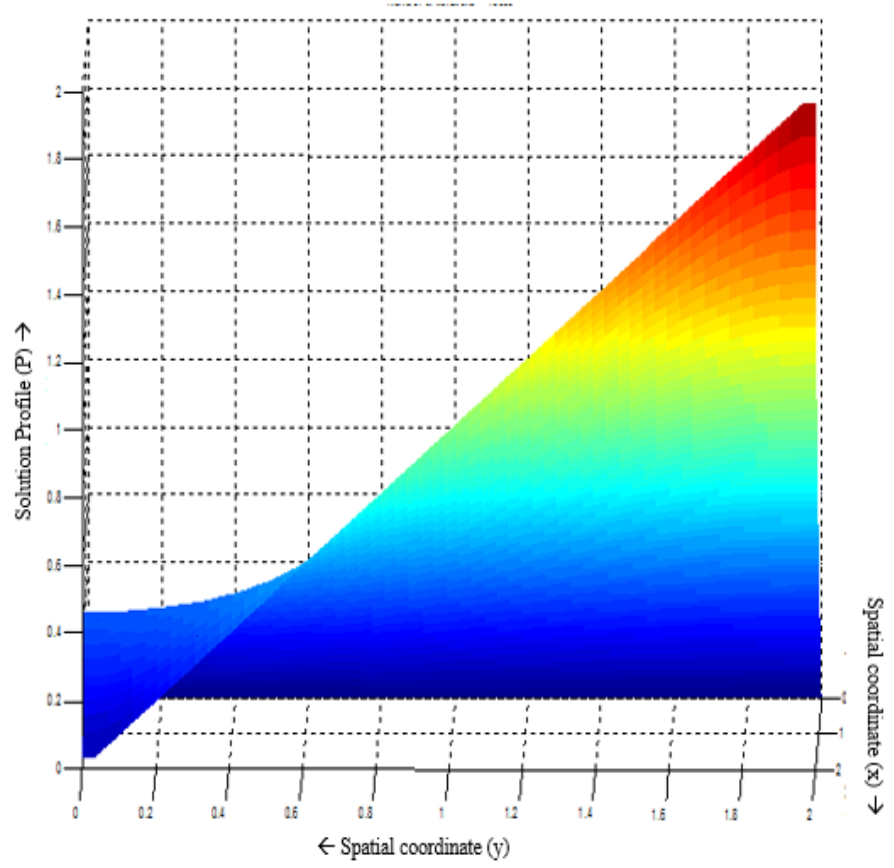

(b) 


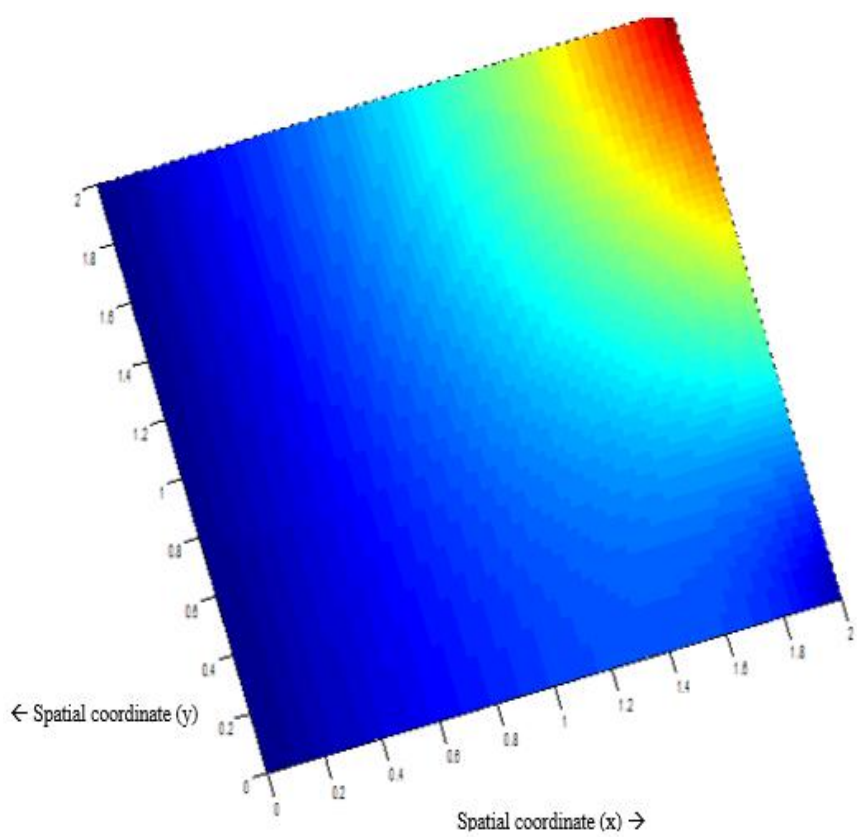

(c)

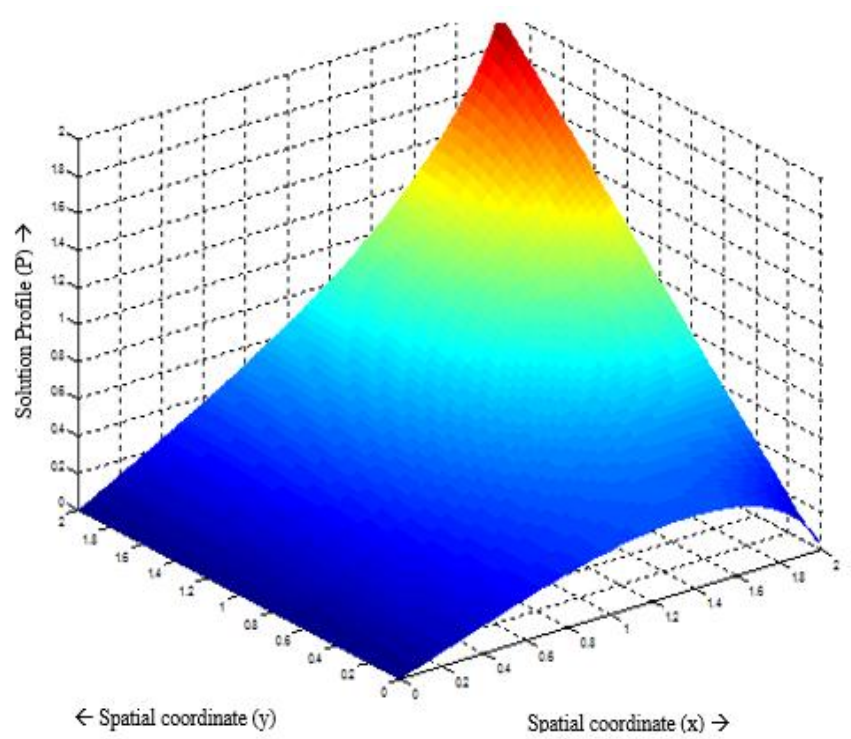

(d)

Fig. 2 (a) and (b) Pressure distribution in $\mathrm{x}$ - and y-direction respectively. (c) shows the top view of the distribution (d) portrays it in $3 \mathrm{D}$

\section{DISCRETIZATION AND NUMERICAL SIMULATION}

Numerical techniques are used to discretize the PDE's with Poisson-elliptic type. Some numerical schemes are governed the algebraic equations in obtaining the approximation solution of Poisson-elliptic equation [6] and [18]. Numerical solutions represent the reservoir behavior are the values of pressure and phase saturation at discrete points in the domain of reservoir at discrete time step. Eq. (1) is solved and compared based on three methods; BEM, FEM and FDM, [16].

\section{A. $B E M$}

The BEM solution is referred from [12]. Eq. (1) can be transformed into an integral equation by multiplying with the free-space Green's function and integrating twice by parts. The free-space Green's function is given as

$G=\frac{1}{2 \pi} \ln \left(\frac{1}{r}\right)$

After the standard manipulation, (1) becomes;

$$
\begin{aligned}
& \alpha p\left(X_{i}, Y_{i}\right)=\frac{1}{2 \pi} \sum_{j=1}^{N} \frac{\partial p}{\partial n_{j}} \int_{s j} \ln \left(\frac{1}{r_{i, j}}\right) d s- \\
& \frac{1}{2 \pi} \sum_{j=1}^{N} p_{j} \int_{s j} \frac{\partial}{\partial n} \ln \left(\frac{1}{r_{i, j}}\right) d s+\frac{1}{2 \pi} \frac{\mu}{k} \sum_{m=1}^{N S S} q_{m} \ln \left(\frac{1}{r_{i, m}}\right)
\end{aligned}
$$

where the reservoir boundary is partitioned into $N$ constant elements with constant properties. Eq. (3) is solved computationally using Fortran programming language.

\section{B. FDM}

Another numerical technique is applied in order to compare with BEM solution [22]. The FDM is used to discretize Eq. (1) by changing partial derivatives with their approximation which is given by:

$$
p_{i, j}=\frac{p_{i+1, j}+p_{i-1, j}+r p_{i, j+1}+r\left(\frac{\mu}{k}\right)}{(2+2 r)}
$$

The system linear of equation developed by Eq. (4) is solved using some iterative schemes such as Jacobi and Gauss Seidel schemes. Matlab software is used as a platform to solve the FDM. The mesh point for Eq. (4) can be visualized in Fig. 3. Table I shows the numerical results for (3) using FDM with Jacobi and Gauss Seidel schemes.

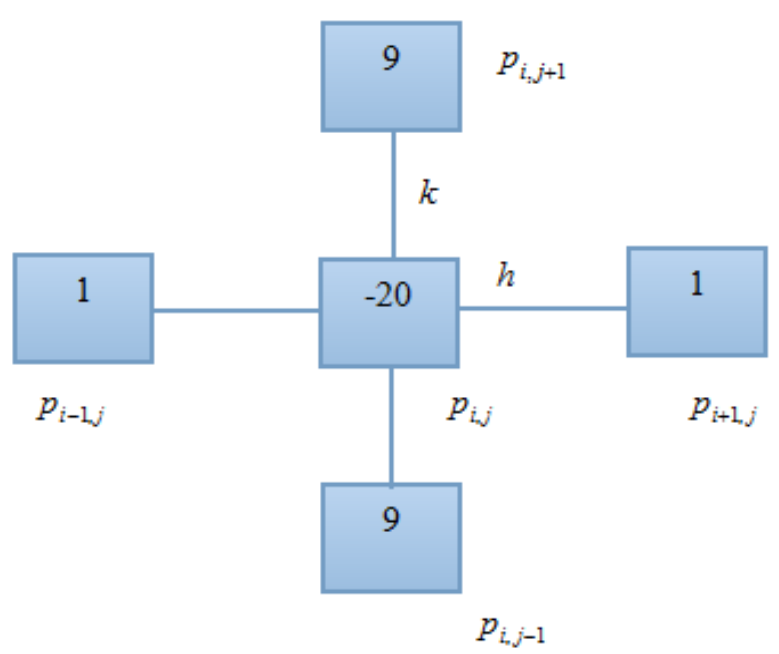

Fig. 3 Structure of Mesh points for FDM discretization 
International Journal of Engineering Research and Technology. ISSN 0974-3154, Volume 13, Number 11 (2020), pp. $3799-3805$

(C) International Research Publication House. https://dx.doi.org/10.37624/IJERT/13.11.2020.3799-3805

TABLE I: Numerical Results for FDM Simulation using Jacobi and Gauss Seidel Schemes

\begin{tabular}{lll}
\hline \hline Scheme & Jacobi & Gauss-Seidel \\
\hline No. of iterations & 17 iterations & 6 iterations \\
Time Execution & 0.0127 seconds & 0.0049 seconds \\
Convergence & Slow & Fast \\
\hline \hline
\end{tabular}

\section{FEM}

Equation (1) is also solved using COMSOL Multiphysics software. This software is based on FEM discretization to solve various engineering and physics applications, especially coupled phenomena or Multiphysics [9]. FEM uses variation technique to minimize an error function and produce a stable solution [7]. FEM encompasses all the techniques for connecting many simple element equations over many smaller sub-domains. The function is to approximate a more complex equation over a larger domain.

The simulation results obtained from these numerical techniques are compared based on the percentage error. Further discussion on the simulation results will be explained in the section IV.

\section{IMPLEMENTATION OF COMSOL MULTIPHYSICS, MATLAB AND FORTRAN}

COMSOL Multiphysics, Matlab and Fortran programming languages are customized and relevant parameters are required for the accurate validation of the mathematical model. The program written in FOTRAN 77 is capable of solving Laplace and Poisson type problems. Basically, this program can be divided into three main parts, namely preprocessor for input data, processor in the part that does the calculations and post-processor for results. In processor, it is divided by four parts that is dimensionless part, form the matrix, solve matrix by using Gaussian elimination and compete for internal points of the oil reservoir.

In COMSOL Multiphysics software, the mathematical modelling was discretized using FEM automatically. In this program, the users need to insert the mathematical modelling manually if the equation not available in the library. The COMSOL simulation environment facilitates all steps in the whole modelling process: defining geometry, specifying physics, meshing, solving, and ends with post processing. MATLAB integrates computation, visualization, and programming is an easy-to-use environment [24]. MATLAB is an interactive system whose basic data element is an array does not require dimensioning. This allows solving many technical computing problems, especially those with matrix and vector formulations, in a fraction of the time it would take to write a program in scalar non-interactive language software. Thus, in this paper, COMSOL Multiphysics software gives the simulation result of FEM while Matlab programming gives the simulation result of FDM. For the validation, the graph of Pressure vs. Depth of oil reservoirs is compared with a graph from FOTRAN programming that developed by [5]. Fig. 2 presents the sequential algorithm for FOTRAN, COMSOL Multiphysics and Matlab software.

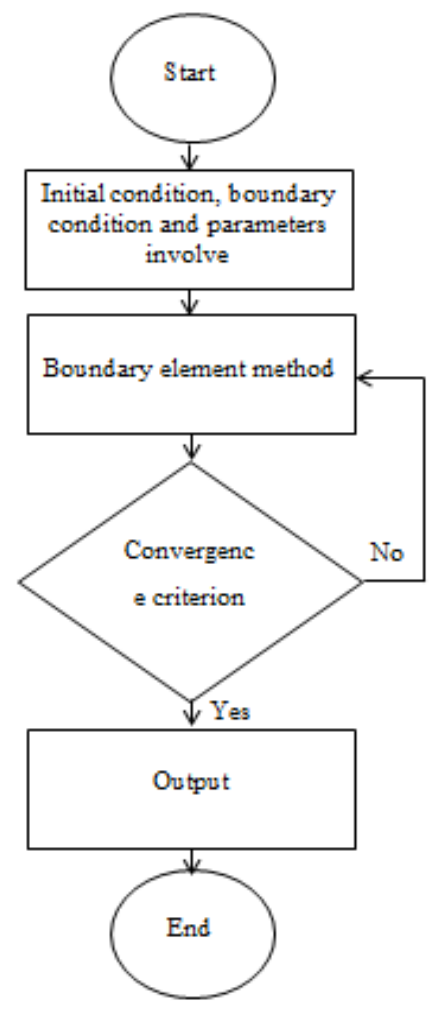

(a)

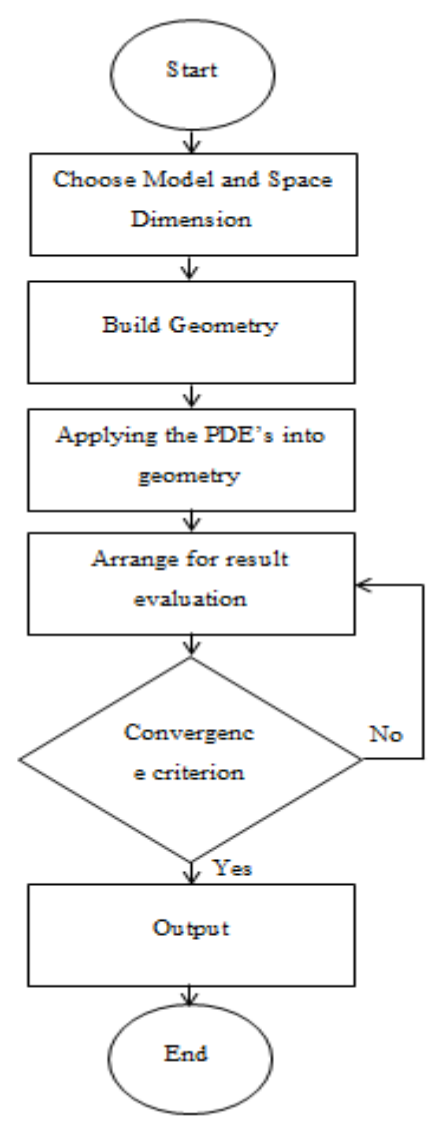

(b) 


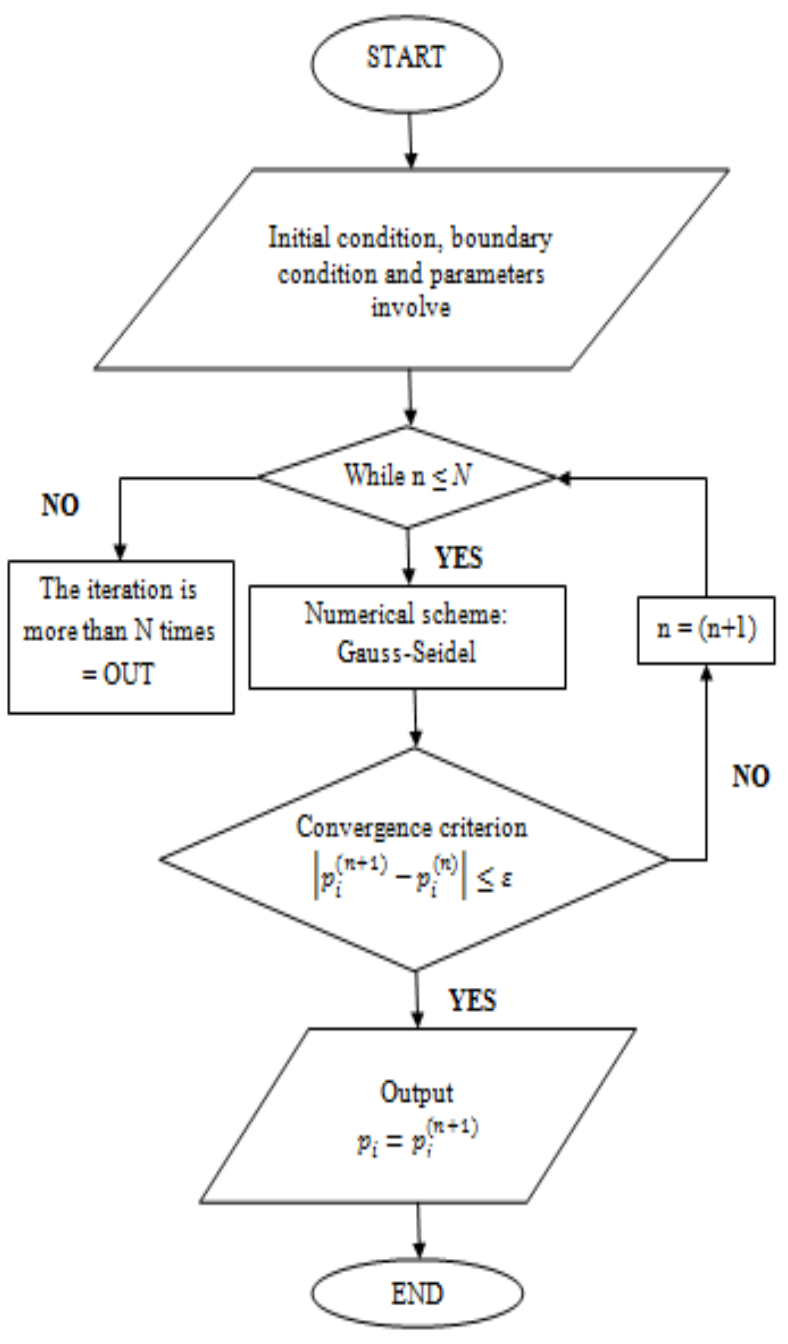

(c)

Fig. 4 Flow chart of the sequential algorithm for (a) Fortran, (b) COMSOL Multiphysics and (c) Matlab software

\section{NUMERICAL RESULTS AND VISUALIZATION}

The differential equation describing the pressure on the productivity of the oil wells model is solved by using the BEM, FEM and FDM. The results of this model give the graph of the pressure limits based on the depth feature. Fig. 3 shows the comparison of the graph of pressure versus level of depth by using three different software. The graph shows that the pressure increase when the level of depth increase. Fig. 3 (a) represents the result of pressure obtained from Fortran software solved by using BEM. Fig. 3 (b) is the COMSOL simulation result by using FEM while Fig. 3(c) presents the Matlab simulation using FDM. The validations of COMSOL and Matlab simulation are compared to the results obtained from Fortran [5]. Based on the simulation from COMSOL and Matlab, it shows that the graph shape have the same pattern with [5].

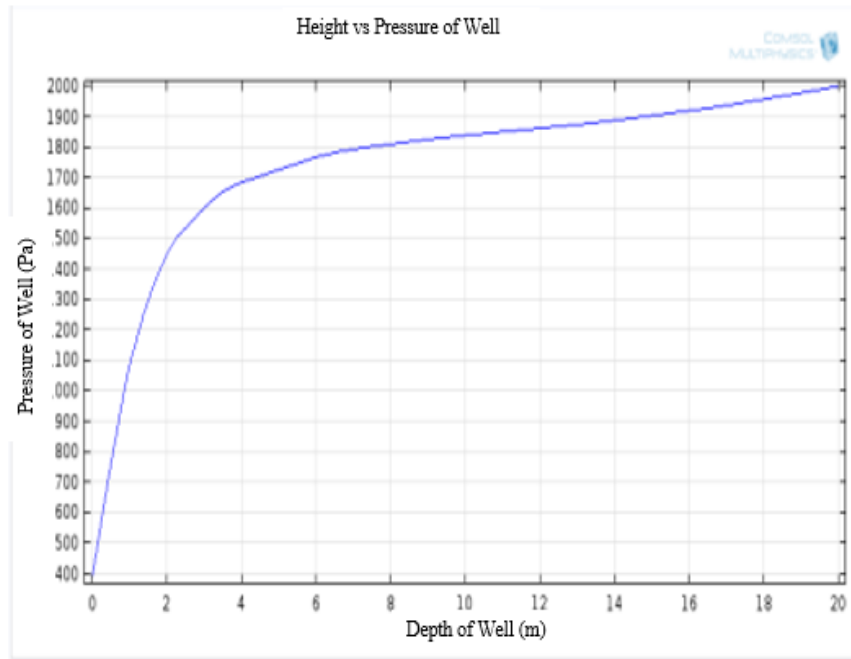

(a)

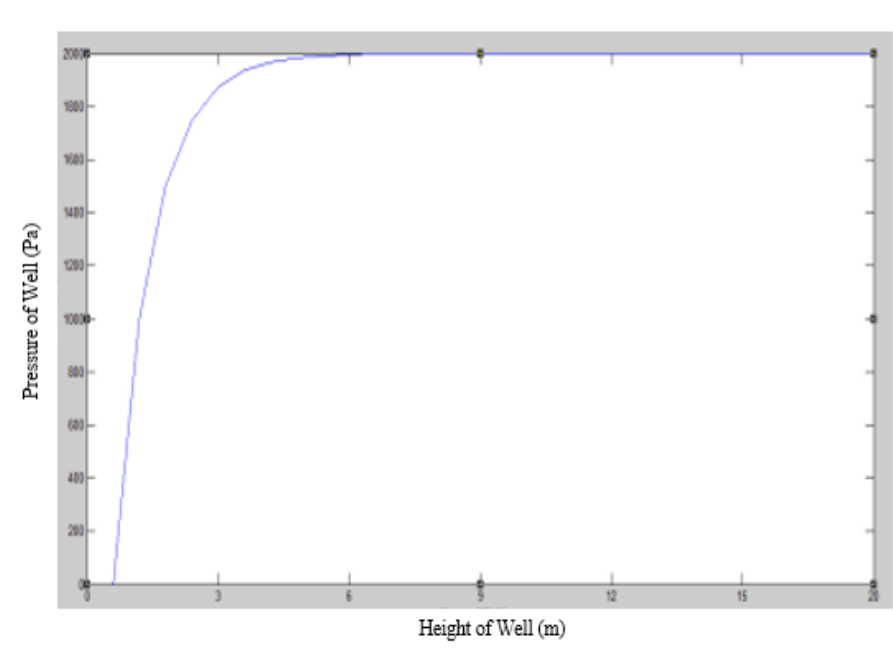

(b)

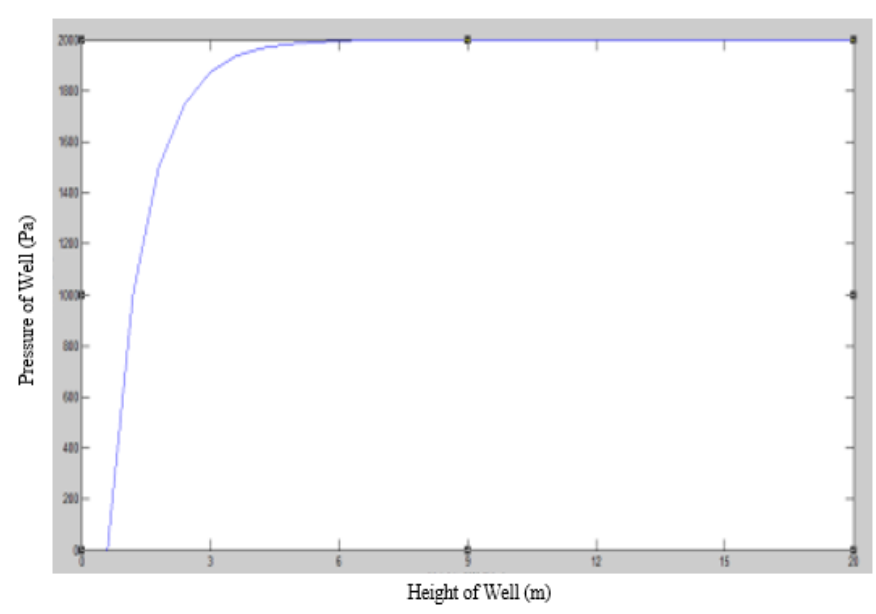

(c)

Fig.5 Graph simulation for Pressure vs. Depth usingthree different software: (a) Fortran, (b) Comsol Multiphysics, and (c) Matlab software 
TABLE II: Percentage Error for COMSOL and Matlab Compared to Fortran Programming Software

\begin{tabular}{cccccc}
\hline \hline$x$ & FOTRAN & COMSOL & $\%$ Error & Matlab & $\%$ Error \\
\hline 0 & 0 & 0 & 0 & 0 & 0 \\
2 & 1200 & 1450 & 0.2083 & 1000 & 0.1670 \\
4 & 1540 & 1680 & 0.0909 & 1750 & 0.1363 \\
6 & 1690 & 1760 & 0.0414 & 1938 & 0.1467 \\
8 & 1800 & 1782 & 0.0100 & 1984 & 0.1022 \\
10 & 1870 & 1830 & 0.0212 & 1996 & 0.0674 \\
12 & 1920 & 1870 & 0.0260 & 2000 & 0.0417 \\
14 & 1960 & 1890 & 0.0357 & 2000 & 0.0204 \\
16 & 1990 & 1920 & 0.0352 & 2000 & 0.0050 \\
18 & 2000 & 1970 & 0.0150 & 2000 & 0.0000 \\
20 & 2000 & 2000 & 0.0000 & 2000 & 0.0000 \\
sum & & & 0.4840 & & 0.6865 \\
\hline \hline
\end{tabular}

The accuracy of the graph is made based on the percentage error which is shown in Table II. Based on the comparison, the summation of percentage error of Matlab is greater than COMSOL, thus, it can be concluded that COMSOL is more superior and closer to FOTRAN compared to Matlab software. This is due to the simulation of COMSOL is based on FEM and the graph visualization of Ibrahim and Shuib (2005) is based on BEM of FEM. Figs. 6 and 7 illustrates the visualization of the oil reservoir and oil well, respectively using COMSOL software. The lower tone of colour refers to the higher pressure. The higher tone refers to the lower pressure. The level of pressure increase when the depth of oil reservoir increases.

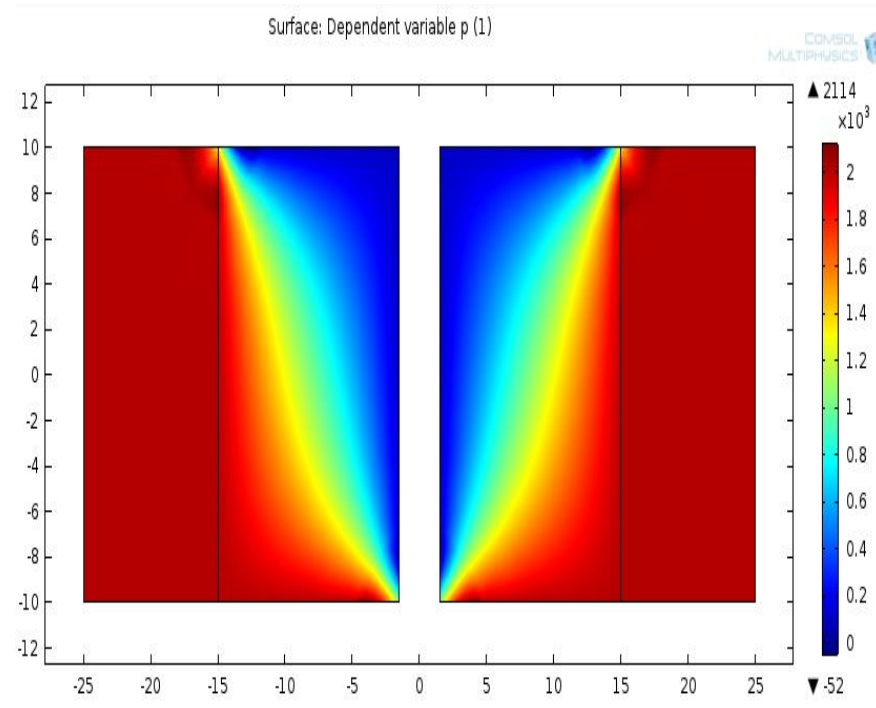

Fig. 6 Visualization of oil reservoir



Fig. 7 Visualization of oil well

\section{CONCLUSION}

As a conclusion, COMSOL Multiphysics, Matlab and Fortran programming are available, relevant and customized to the specific requirements. The visualization and post processing of COMSOL Multiphysics is high potential when dealing with a multiple leases reservoir. COMSOL Multiphysics offers the advanced visualization technique especially in targeted arbitrarily shaped of oil well reservoir. Meanwhile, Matlab is relevant to program the sequential algorithm of modelling because of the simplicity, straight forward and situationdependence software. High-level language for numerical computation is well suited for capturing the visualization. According to the performance comparison, the programming structured of Fortran is the alternative software for predicting the reservoir pressure based on BEM discretization.

\section{ACKNOWLEDGMENT}

The author would like to acknowledge the support from the Research Acculturation Collaboration Effort (RACE) under a grant number of RACE/F2/SG/UNIMAP/5 from the Ministry of Higher Education Malaysia.

\section{REFERENCES}

[1] Gainville, M., Roux, P., Pons, M., Pons, M., Ricordeau, A., Paen, D. (2007). Integrated Flow Modeling Platform Using CAPE-OPEN Standard. SPE Annual Technical Conference and Exhibition 2007, ATCE 2007; Anaheim, CA; United States, 6, pp. 3977-3986.W.-K. Chen, Linear Networks and Systems (Book style). Belmont, CA: Wadsworth, 1993, pp. 123-135.

[2] Ghalambor, A. and Guo, B. (2010). How Significant is the Formation Damage in Multi-Fractured Horizontal Well. SPE International Symposium and Exhibition on formation Damage Control, 10-12 Feb, Louisiana, USA, 
International Journal of Engineering Research and Technology. ISSN 0974-3154, Volume 13, Number 11 (2020), pp. 3799-3805

(C) International Research Publication House. https://dx.doi.org/10.37624/IJERT/13.11.2020.3799-3805

SPE-125905-MS.

[3] Hagoort, J. (2009). A Simplified Analytical Method for Estimating the Productivity of A Horizontal Well. Journal of Petroleum Science and Engineering, 64 (1), 77-87.

[4] Huimei, G., Yingfu, H., Hanqiao, J. and Minfeng, C. (2007). Pressure Transient Analysis of Arbitrarily Shaped Fractured Reservoirs. Petroleum Science, 4, pp. 66-70.

[5] Ibrahim, M. N. and Shuib, S. (2005). Study On Oil Reservoir Productivity Performance Via Combination of Taguchi and BEM Analyses. Electronic Journal of Boundary Element , 3, 1-13.

[6] Pourabdollah, K. and Mokhtari, B. (2012). Well Surveillance Using Multivariate Thermal Measurement. Journal of Thermal Analysis and Calorimetry, 107, 1353-1361.

[7] Reddy, J. N. (2006). An Introduction to the Finite Element Method. Mc Graw Hill, New York.

[8] Ibrahim, M.N.M., Abdullah, J., Mahmud, A.S. (2003). Number of Boundary Element Effects on the Productivity Performance of An Oil Reservoir. Boundary Elements, 4, pp. 259-265

[9] Li, Q., Ito, K., Wu, Z., Lowry, C.S. and Loheide II, S.P. (2009). COMSOL Multiphysics: A Novel Approach to Ground Water Modeling. Groundwater, 47 (4), 480-487.

[10] Mavor, M.J. and Cinco-Ley, H. (1979) Transient Pressure Behavior of Naturally Fractured Reservoirs. SPE, 7977.

[11] Ibrahim, M.N., Numbere, D.T. (1999) Boundary Element Analysis of the Productivity of Complex Petroleum Well Configurations. Boundary Element Technology XIII. Proceedings of the 1999 Thirteenth International Conference on Boundary Element Technology, BETECH '99, pp. 25-34.

[12] Ibrahim, M.N. (2004). Prediction of Oil Production of Individual Operator in A Multiple Leases Reservoir. In Advances in Boundary Element Technology V, Editors: V. M. A. Leitao and M. H. Aliabadi, EC Ltd., United Kingdom, pp. 121-125.

[13] Sargent, R.G. (2005). Verification and Validation of Simulation Models. Proceeding WSC '05 Proceedings of the 37th Conference on Winter Simulation, 130-143.

[14] Zhang, W.M. and Zeng, P. (1992) A Boundary Element Method Applied to Pressure Transient Analysis of Irregularly Shaped Double Porosity Reservoirs. SPE, 25284.

[15] Yildirim, S. , Exact and Numerical Solutions of Poisson Equation for Electrostatic Potential Problems, Mathematical Problems in Engineering, Volume 2008, 11 pages

[16] Gockenbach, M.S. ,Partial Differential Equations Analytical and Numerical Methods, 2nd Edition; Michigan Technological University; Houghton, Michigan; 2011.Michigan Technological University;
Houghton, Michigan; 2011.

[17] Sauter, S.A., Christoph, S., Boundary Element Methods, Springer 2011.

[18] Dong Guang, Deng Jingen, Zhu Haiyan, ThreeDimensional Numerical Calculation Analysis Of Stress Induced By Pore Pressure Variation, Journal of Theoretical and Applied Information Technology, December 2012, Vol. 46 No.1.

[19] Zhang Lie-Hui; Li Cheng-Yong; Liu Qi-Guo; Li Yun, Application of boundary element method to fluid flow into horizontal wells in complex boundary reservoirs, Chinese Journal of Computational Mechanics Year 2009, Issue 2, Page 287-290.

[20] Alias, N, Mustaffa, M.N, Saipol, H.F, Ghani, A.C.A, High performance large sparse PDEs with parabolic and elliptic types using AGE method on DPCS, Advanced Science Letters, 20, Vol 10-12, 2014, pg:1956-1960

[21] Alias, N., Saipol, H.F.S., Ghani, A.C.A.,Mustaffa, M.N., Embedded system for high performance digital temperature sensor on distributed parallel computer system, Advanced Science Letters, 2014, 20 (2), pp. 406-411

[22] Azziz, N. H. A. N. H. A., Muhamad, W. Z. A. W. W. Z. A. W., \& Zain, N. A. M. N. A. M. (2017). WebBased Numerical Algorithm Library : A Review. International Journal of Applied Engineering Research, 12(20), 10068-10072.

[23] Junoh, A. K., Nopiah, Z. M., Muhamad, W. Z. A. W., \& Nor, M. J. M. (2011). Multi Objective Optimization of Noise and Vibration in Passenger Car Cabin by Using Goal Programming Approach. Advanced Materials Research, 383-390, 976-983.

[24] Muhamad, W. Z. A. W., Jamaludin, K. R., Saad, S. A., Yahya, Z. R., \& Zakaria, S. A. (2018). Random binary search algorithm based feature selection in Mahalanobis Taguchi system for breast cancer diagnosis. AIP Conference Proceedings, 1974(1), 020027. 